\title{
BMJ Open Developing a training programme in physical activity counselling for undergraduate medical curricula: a nationwide Delphi study
}

\author{
Apichai Wattanapisit, ${ }^{\oplus 1,2}$ Prachyapan Petchuay, ${ }^{1}$ Sanhapan Wattanapisit, ${ }^{3}$ \\ Titiporn Tuangratananon ${ }^{4}$
}

To cite: Wattanapisit A, Petchuay P, Wattanapisit S, et al. Developing a training programme in physical activity counselling for undergraduate medical curricula: a nationwide Delphi study. BMJ Open 2019;9:e030425. doi:10.1136/ bmjopen-2019-030425

- Prepublication history for this paper is available online. To view these files, please visit the journal online (http://dx.doi. org/10.1136/bmjopen-2019030425).

Received 13 March 2019 Revised 05 August 2019 Accepted 12 August 2019

A) Check for updates

(c) Author(s) (or their employer(s)) 2019. Re-use permitted under CC BY-NC. No commercial re-use. See rights and permissions. Published by BMJ.

${ }^{1}$ Department of Clinical Medicine, Walailak University School of Medicine, Thasala, Nakhon Si Thammarat, Thailand ${ }^{2}$ Family Medicine Clinic, Walailak University Hospital, Thasala,

Nakhon Si Thammarat, Thailand

${ }^{3}$ Family Medicine Unit, Thasala Hospital, Thasala, Nakhon Si

Thammarat, Thailand

${ }^{4}$ International Health Policy

Program, Thailand, Ministry

of Public Health, Muang,

Nonthaburi, Thailand

Correspondence to Dr Apichai Wattanapisit; apichai.wa@wu.ac.th

\section{ABSTRACT}

Objectives To identify the essential content and approaches for developing a training programme in physical activity $(\mathrm{PA})$ counselling for undergraduate medical curricula.

Design A three-round Delphi survey was conducted to investigate four key topics: (1) contents of PA counselling in medical education; (2) teaching and learning methods; (3) medical school collaboration and (4) educational policy implementation. Round 1 collected opinions from the participants. Round 2 focused on scoring the opinions. Round 3 summarised the expert opinions. A mean score of 4 or above identified as an important item.

Setting All 23 medical schools in Thailand.

Participants Academic staff who were experts or in charge of medical schools in the fields of PA, health promotion or medical education.

Results A total of 20 representatives from 18 of the 23 Thai medical schools participated in the study (for a response rate of $78.2 \%$ ). The top three most important indicators of knowledge were (1) the definition and types of PA (4.75 \pm 0.55$)$, (2) the FITT principle (frequency, intensity, time and type) $(4.75 \pm 0.55)$ and (3) the benefits of PA (4.65 \pm 0.67$)$. The most important component of the training involved general communication skills (4.55 \pm 0.60$)$. An extracurricular module ( $4.05 \pm 0.76)$ was preferable to an intracurricular module $(3.95 \pm 0.94)$. Collaborations with medical education centres and teaching hospitals $(4.45 \pm 0.78)$ and supporting policies to increase medical students' $\mathrm{PA}(4.40 \pm 0.73)$ were considered to be important.

Conclusion Knowledge and counselling skills are important for PA counselling. Building collaborations between medical education and health institutions, as well as implementing effective educational policies, are key approaches to the integration of PA counselling into medical education. Future research should focus on investigating the effects of training in PA counselling on the learning outcomes of medical students and the clinical outcomes of patients.

\section{INTRODUCTION}

Promoting physical activity (PA) in clinical settings is a promising approach to the reduction of physical inactivity. ${ }^{1}$ From the
Strengths and limitations of this study

- The study had a $78.2 \%$ response rate (18 out of 23 medical schools) from the medical schools in Thailand.

- The characteristics of the Delphi study, using a series of questionnaires, helped to achieve the consensus of expert opinion and avoid problems arising from a few powerful participants and group pressures.

- The study sites did not include medical education centres and teaching hospitals, which were affiliated with the medical schools or universities.

patients' point of view, physicians are credible sources of health-related information and guidance. ${ }^{2}$ PA counselling is an effective strategy to increase the PA level of patients. ${ }^{3}$ A systematic review concluded that 1 out of 12 sedentary patients will become physically active after the promotion of PA in healthcare services. ${ }^{4}$ PA can reduce risks of mortality and cardiovascular disease. ${ }^{5} 6$ This leads to the concept that exercise and PA are important aspects of lifestyle in medicine. ${ }^{7-9}$ However, important barriers to PA counselling include time constraints and a lack of knowledge and communication skills among healthcare professionals. ${ }^{10}$ The WHO proposes a policy action to strengthen curricula of medical professionals to ensure effective integration of the health benefits of PA into the formal training. ${ }^{11}$

Inadequate training in PA topics may be one barrier to PA counselling. Medical curricula address a limited number of PA topics during undergraduate training, representing only 4.2 hours in the UK, 8.1 hours in the USA and 12.3 hours in Australia. ${ }^{12-14}$ Given the time spent teaching PA topics, overall training in PA counselling is insufficient. A study conducted in Thailand found that during the 6-year curriculum, even when the curriculum 
included 25 hours of instruction in PA topics, the training specifically addressing PA counselling is absent. ${ }^{15}$ Thus, training in PA counselling is still limited at both undergraduate and postgraduate levels. ${ }^{1617}$

Given the concept that 'exercise is medicine', exercise or PA should be regarded as an important aspect of treatment for every patient. ${ }^{18}$ The UK approach, Making Every Contact Count, encourages healthcare professionals to talk with patients about their health and wellbeing during routine interactions. ${ }^{19}$ According to these concepts, training in PA counselling can be instilled as a component of the patient care programme for medical students. The gap in knowledge involves how to best develop medical students' awareness and abilities to conduct PA counselling during their clinical rotations and ultimately in their postgraduate clinical practices. As a result, this study was designed to identify the essential content and approaches for developing effective techniques that can be incorporated into the undergraduate medical curricula for PA counselling.

\section{METHODS}

\section{Study design}

A Delphi survey method was conducted to gather opinions from expert medical educators in Thailand. The initial questions emerged from a roundtable discussion. A panel of experts, composed of eight specialist academics and practitioners from six institutions, was formed in July 2018; these experts were from the fields of PA, medical education, primary care, sports science, sports medicine, internal medicine, endocrinology, orthopaedics, as well as health promotion and health policy. This panel addressed four key topics: (1) the content of PA counselling in medical education; (2) teaching and learning methods; (3) medical school collaborations and (4) educational policy implementation.

The authors then conducted a three-round survey through online questionnaires. Round 1 was designed to collect additional opinions from the participants, round 2 focused on scoring the opinions and round 3 entailed producing a summary of the expert opinions.

\section{Setting and participants}

The authors sent letters to contact the deans of all 23 medical schools in Thailand to request the permission to conduct the study and the names of one to two eligible participants. The inclusion criterion was academic staff who were experts or in charge of medical schools in the fields of PA, health promotion or medical education. Medical school representatives who had participated in the initial roundtable discussion were excluded.

\section{Data collection and analysis}

The online questionnaires were sent to the participants' email addresses. Each round took about 1 month. If there was no response to the questionnaires within 2 weeks, the authors would send an email to remind the participants. The non-responders would be prompted again by a phone call 1 week after the second email. After that prompt, any non-responders were dropped from the study and no longer considered a potential source of data. After the completion of each round, the research team analysed data and developed the questions for the next rounds. Participants' names and institutions were coded to ensure confidentiality and anonymity. All data collection was conducted between August and December 2018. The study protocol was approved by the Human Research Ethics Committee of Walailak University (protocol number: WUEC-18-039-01).

\section{Round 1}

The questionnaire, using Google Forms (Alphabet, Mountain View, California, USA), provided some items that emerged from the roundtable discussion of the four key topics. The authors asked the medical school representatives to include additional opinions on each key topic using open-ended questions. Additionally, the authors offered participants to include their opinions to form additional key topics. The answers from round 1 were qualitatively analysed by the authors and confirmed by discussion within the research team. All the items that emerged after the round 1 analysis were collected as questions for round 2.

\section{Round 2}

The questionnaire, using Google Forms, was developed based on analysis of round 1 . The authors asked the participants to score each item from 1 to 5 based on their opinions, in which a score of $1=$ not important; $2=$ slightly important; $3=$ moderately important; $4=$ important and $5=$ very important. The overall score for each item was calculated as a mean score with a SD. These calculations were performed using Excel software (a component of the Office 365 University package, Microsoft, Redmond, Washington, USA). Achieving consensus was considered by having $75 \%$ of participants' scores within two categories-important (score $=4$ ) and very important $($ score $=5) .{ }^{20}$ The data analysed from round 2 were ranked by mean scores and developed into the round 3 questionnaire.

\section{Round 3}

The items provided in the questionnaire, using Google Sheets (Alphabet, Mountain View, California, USA), were similar to the items provided in the round 2 questionnaire. However, in this round, the participants could see the sequences of items ranked by mean scores, item mean scores and SDs from the results of round 2, as well as their previous scores. In this final round, the participants were asked to confirm or change their scores. The final consensus criterion was defined as $75 \%$ of agreement among participants. ${ }^{20}$ The consensus in this round meant that the item was considerd important or very important by at least $75 \%$ of experts. The mean scores and SDs were also calculated in this round. The items with mean 


\begin{tabular}{|c|c|c|c|c|}
\hline Participant code & Institution code & Gender & Age (years) & Specialty \\
\hline P1 & 11 & Female & 34 & Family medicine \\
\hline P3 & 13 & Female & 39 & Physiology \\
\hline P4 & 14 & Male & 42 & Physical medicine and rehabilitation \\
\hline P7 & 16 & Female & 33 & Paediatrics \\
\hline P8 & 17 & Male & 53 & Public health/epidemiology \\
\hline P9 & 18 & Female & 34 & Family medicine \\
\hline P10 & 19 & Female & 48 & Public health/health economics \\
\hline P14 & 113 & Male & 43 & Internal medicine \\
\hline P15 & 114 & Male & 38 & Family medicine \\
\hline P16 & 114 & Male & 48 & Physical medicine and rehabilitation \\
\hline P17 & 115 & Female & 40 & Family medicine \\
\hline P18 & 116 & Male & 30 & Family medicine \\
\hline P19 & 117 & Male & 39 & Epidemiology \\
\hline P20 & 118 & Female & 33 & Family medicine \\
\hline
\end{tabular}

scores $\geq 4$ were considered to be important. The mean scores reflected the level of importance of items.

\section{Patient and public involvement}

The study did not involve patients as study participants. The presentation of the findings was anonymous to preserve confidentiality.

\section{RESULTS}

\section{Baseline characteristics of participants}

A total of 20 representatives from 18 of the 23 Thai medical schools, including both public and private institutions, participated, for a response rate of $78.2 \%$. The participants were aged between 30 and 61 years (mean $40.5 \pm 8.2$ years). A total of $11(55.0 \%)$ were females and $10(50 \%)$ specialised in family medicine (table 1$)$. All participants were active throughout all the three rounds of the survey.

\section{Content of PA counselling in medical education}

Knowledge of PA and training in counselling were the two sub-topics regarding contents of PA counselling in medical education. In round 1 , the items regarding knowledge of PA emerged from 7 to 23 items, and the items regarding training in counselling increased from 3 to 10 items. The top three most important areas of knowledge were (1) the definition and types of PA $(4.75 \pm 0.55)$, (2) the FITT principle (frequency, intensity, time and type) $(4.75 \pm 0.55)$ and (3) the benefits of PA $(4.65 \pm 0.67)$. The top three most important topics for training the students in counselling were (1) general communication skills $(4.55 \pm 0.60),(2)$ counselling techniques $(4.45 \pm 0.69)$ and (3) the 5 A's (ask, advise, assess, assist and arrange) $(4.30 \pm 0.73)$ (table 2$)$.

\section{Teaching and learning methods}

Several items regarding intracurricular activities, extracurricular activities and evaluation were raised by the participants in round 1. There was a preference for training in PA counselling to be provided as an extracurricular module $(4.05 \pm 0.76)$ rather than an intracurricular module $(3.95 \pm 0.94)$. The top priorities for the intracurricular activities were (1) interactive activities $(4.50 \pm 0.69)$, (2) interprofessional education

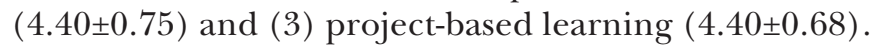
The most important extracurricular activities were online modules $(4.35 \pm 0.67)$, followed by interactive activities $(4.30 \pm 0.73)$ and interprofessional education $(4.30 \pm 0.66)$. The important evaluation methods included (1) self-assessment and reflection (4.40 \pm 0.68$)$, (2) student behaviours $(4.20 \pm 1.11)$ and (3) clinical performance of students $(4.20 \pm 0.77)$ (table 3$)$.

\section{Medical school collaborations}

According to the initial survey, collaboration among organisations was a key to successful implementation of training in PA counselling. There were two organisations mentioned in the roundtable discussion. Subsequently, 12 additional items were offered during round 1. According to the final results, medical schools should collaborate 
Table 2 Content of physical activity counselling in medical education

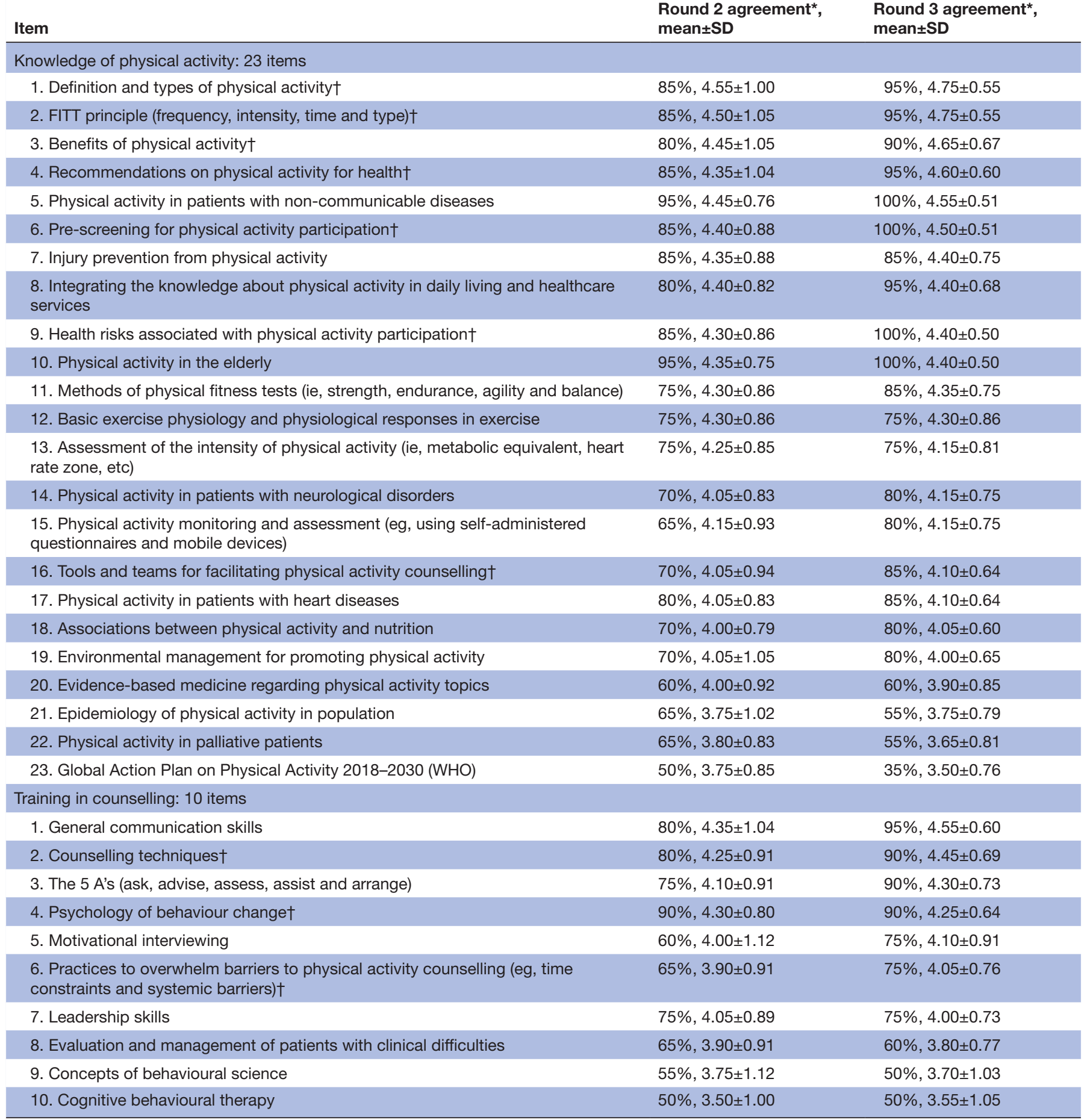

${ }^{*}$ Percentage of participants who scored 4 and 5 for each item. †ltem emerged from the roundtable discussion.

with (1) medical education centres and teaching hospitals $(4.45 \pm 0.78),(2)$ the Society of Medical Students of Thailand $(4.39 \pm 0.72)$ and (3) Thai Health Promotion Foundation (4.38 \pm 0.74$)$ (table 4).

\section{Educational policy implementation}

The top three ranks of educational policy implementation were (1) support policies to increase medical

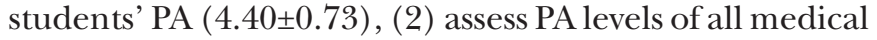

students $(4.36 \pm 0.85)$ and (3) provide active environments $(4.35 \pm 0.79)$ (table 5).

\section{DISCUSSION}

The nationwide survey that was performed in this study gave insight into the key elements that are important for PA counselling in medical education, teaching and learning methods, medical school collaboration and educational 
Table 3 Teaching and learning methods

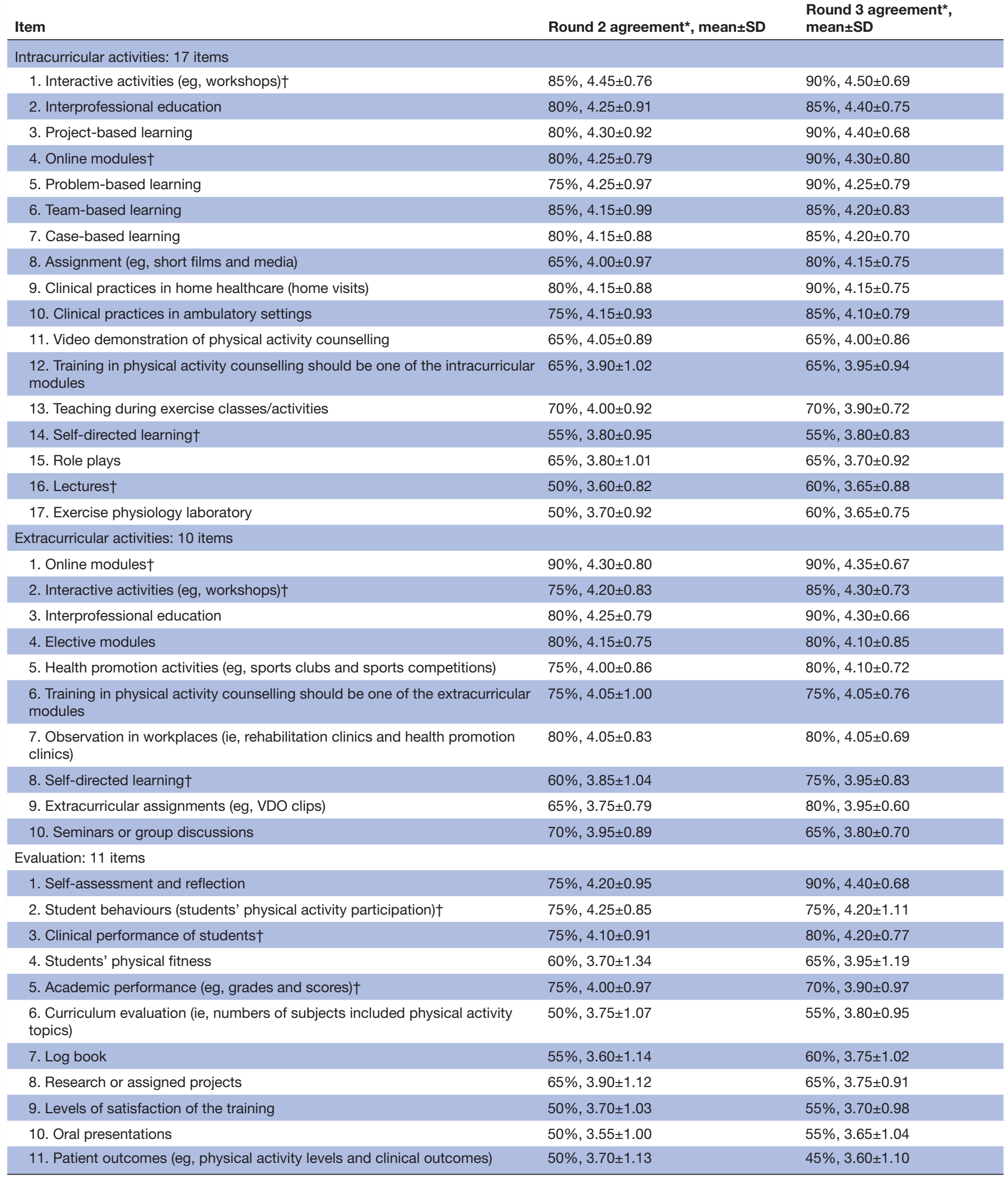

*Percentage of participants who scored 4 and 5 for each item. †ltem emerged from the roundtable discussion.

policy implementation. The definition and types of PA and general communication skills were the most important contents of PA knowledge and training in counselling.
Training in PA counselling could use either intracurricular or extracurricular modules. Interactive activities, such as workshops, were the most important intracurricular 
Table 4 Medical school collaborations

Round 3

Round 2 agreement*, agreement*,

\begin{tabular}{|c|c|c|}
\hline Item & mean $\pm S D$ & mean $\pm S D$ \\
\hline \multicolumn{3}{|l|}{ Medical school collaborations: 14 items } \\
\hline 1. Medical education centres and teaching hospitals & $80 \%, 4.40 \pm 0.94$ & $85 \%, 4.45 \pm 0.78$ \\
\hline 3. Thai Health Promotion Foundation & $80 \%, 4.30 \pm 0.91$ & $90 \%, 4.38 \pm 0.74$ \\
\hline 4. Collaborative Project to Increase Production of Rural Doctor & $70 \%, 4.15 \pm 0.97$ & $85 \%, 4.36 \pm 0.76$ \\
\hline 6. Networks of university health promotion (university levels - within a country or regions) & $75 \%, 4.15 \pm 0.94$ & $80 \%, 4.29 \pm 0.84$ \\
\hline 7. Consortium of Thai Medical Schools & $75 \%, 4.15 \pm 0.94$ & $90 \%, 4.29 \pm 0.71$ \\
\hline 8. Ministry of Public Health & $60 \%, 3.85 \pm 1.02$ & $65 \%, 4.08 \pm 0.90$ \\
\hline 9. Institute of Physical Education & $60 \%, 3.80 \pm 0.98$ & $65 \%, 3.95 \pm 0.92$ \\
\hline $\begin{array}{l}\text { 10. Introduce the physical activity research collaboration among Thai medical schools via } \\
\text { Medical Research Network of the Consortium of the Thai Medical schools (MedResNet) }\end{array}$ & $65 \%, 3.80 \pm 0.94$ & $60 \%, 3.91 \pm 0.88$ \\
\hline 12. Conduct surveys on physical activity among Thai medical students & $65 \%, 3.80 \pm 1.04$ & $65 \%, 3.86 \pm 0.90$ \\
\hline 13. University Sports Board of Thailand & $60 \%, 3.85 \pm 0.91$ & $60 \%, 3.85 \pm 0.91$ \\
\hline 14. International Federation of Medical Students' Association-Thailand & $50 \%, 3.65 \pm 1.06$ & $55 \%, 3.68 \pm 1.06$ \\
\hline
\end{tabular}

${ }^{*}$ Percentage of participants who scored 4 and 5 for each item.

†ttem emerged from the roundtable discussion.

activities, while online modules were the most meaningful extracurricular activities. The emphasis on evaluation was self-assessment and reflection by medical students. To drive the training in PA counselling in medical education, collaboration among institutions was needed, including good collaboration between medical education centres and teaching hospitals. Policies to increase the awareness of medical students about the importance of PA for health had the highest priority.

Overall, the participants recognised about two-thirds of the items as being important (with a mean score of $\geq 4$ ). This reflected the importance of training in PA counselling in medical education. The evidence from the study supported the concept that training in PA counselling

Table 5 Educational policy implementation

\begin{tabular}{|c|c|c|}
\hline Item & $\begin{array}{l}\text { Round } 2 \text { agreement } \\
\text { mean } \pm \text { SD }\end{array}$ & $\begin{array}{l}\text { Round } 3 \text { agreement*, } \\
\text { mean } \pm \text { SD }\end{array}$ \\
\hline \multicolumn{3}{|l|}{ Educational policy implementation: 10 items } \\
\hline $\begin{array}{l}\text { 1. Support policies to increase medical students' physical activity (eg, leisure time and } \\
\text { healthy behaviours) }{ }^{\dagger}\end{array}$ & $80 \%, 4.25 \pm 1.04$ & $85 \%, 4.40 \pm 0.73$ \\
\hline 2. Assess physical activity levels of all medical students (ie, annual check-ups) & $80 \%, 4.25 \pm 1.00$ & $85 \%, 4.36 \pm 0.85$ \\
\hline $\begin{array}{l}\text { 3. Provide active environments (eg, walkable areas, bicycle lanes and parks, and } \\
\text { recreational areas) }\end{array}$ & $80 \%, 4.25 \pm 1.04$ & $80 \%, 4.35 \pm 0.79$ \\
\hline 5. Promote medical students' physical activity ${ }^{\dagger}$ & $85 \%, 4.25 \pm 0.83$ & $90 \%, 4.34 \pm 0.66$ \\
\hline $\begin{array}{l}\text { 6. Integrate physical activity topics and counselling in preclinical and clinical subjects } \\
\text { (educational policies) }\end{array}$ & $85 \%, 4.25 \pm 0.95$ & $80 \%, 4.23 \pm 0.89$ \\
\hline 7. Promote role models (eg, medical teachers and residents) & $65 \%, 3.85 \pm 0.99$ & $70 \%, 4.04 \pm 0.91$ \\
\hline
\end{tabular}

${ }^{*}$ Percentage of participants who scored 4 and 5 for each item.

†ltem emerged from the roundtable discussion. 
during medical education improved the knowledge, skills and attitudes of the medical students. ${ }^{16}$ Trained physicians were more likely than untrained physicians to discuss PA benefits. ${ }^{21}$ Although lifestyle aspects of medicine were deemed important, the majority of medical schools did not include the topic of lifestyles and PA in their curricula. ${ }^{22}$ One possible reason was that no structured models to integrate lifestyle medicine topics into medical education were available. ${ }^{23}$ The findings that emerged from this study could help to develop future educational programmes. Another possible explanation of the lack of training in PA counselling was the inadequacy of awareness. Stakeholders should educate medical schools, universities, academics and students to regard PA as an important factor for health maintenance and inactivity as a risk factor for the development of illnesses. Additionally, stakeholders should reinforce knowledge, capacity and skills in the promotion of PA through training programmes and opportunities. ${ }^{11}$

The findings of the present study revealed many considerations for the development of training in PA counselling in medical education. Accordingly, in terms of medical education, designing an appropriate programme of learning was necessary. Many items were considered to be important for knowledge and necessary for the acquisition of good counselling skills. Therefore, the newly designed programmes should focus on the knowledge base of the learners and the baseline training needed for most of the medical students, who are non-specialists. One challenge for inserting any new topic into the medical curriculum is the problem of curriculum overload. Assuring appropriate content and sequences of the topics presented to the medical students is necessary to ease their academic burden and avoid information overload. ${ }^{24}$ Moreover, a well-designed PA module with a limited duration ( 1.5 hours) can improve the competence in counselling of medical students. ${ }^{25}$ To achieve the learning outcomes that are necessary to meet the students' PA counselling needs, it must be recognised that competent communication with patients will require specific knowledge and skills, including (1) content-what to do with communication reactions, (2) process-how communication interactions occur and (3) perceptual skills-how that impacts communication, including feelings, thoughts and attitudes. ${ }^{26}$ This can be achieved using several teaching, learning and evaluation methods. ${ }^{27}{ }^{28}$ According to the results of the study, the important evaluation methods covered three domains, including knowledge of PA, PA behaviours and clinical skills.

An emerging key topic involved building collaborations among medical schools and other organisations. These collaborations can increase opportunities for developing faculty, sharing resources across institutions and enriching the combination of the knowledge, innovation and experiences. ${ }^{29}$ Important items for educational policy implementation included 7 out of the 10 policies recognised as helpful to increase PA in medical students. Previous evidence supported a strong association between medical students' PA behaviours and PA counselling attitudes and practices. ${ }^{30}{ }^{31}$ In other words, physically active medical students are more likely to counsel their patients about PA. This is a notion to develop PA counselling via promoting PA behaviours among medical students. Advocating for an educational policy to improve the PA behaviours of medical students requires a specific understanding of medical students' initial PA behaviours, as well as facilitating and barrier factors for PA. ${ }^{32}$

\section{Strengths and limitations of the study}

There were some strengths of the study. First, the study had a $78.2 \%$ response rate (18 out of 23 medical schools) from the medical schools in a national-level survey. Moreover, all participants remained engaged throughout the study. A strategy used in the study to maintain the participation was sending extra prompts with specific reminder content. ${ }^{33}$ Second, the characteristics of the Delphi study, using a series of questionnaires, helped to achieve the consensus of expert opinion and avoid problems arising from a few powerful participants and group pressures. ${ }^{3435}$ Third, although the findings did not provide a ready-to-use PA counselling module, they did lead to more specific components of PA education and training than are available with the previous non-specific components provided by the Medical Council of Thailand. ${ }^{36}$ The findings also are adaptable and transferable from the national level in the Thai context to the international level for developing a new approach to this aspect of medical education.

Three limitations of the study were identified. The study sites did not include medical education centres and teaching hospitals, which were affiliated with the medical schools or universities. However, medical education centres and teaching hospitals were identified as a potential part of a collaboration to develop the training in PA counselling. Another limitation was that half of the participants specialised in family medicine, which might be skewed. It might be useful to include more sport and exercise specialists in this survey. A reason that could explain this result because training in non-communicable diseases, lifestyle medicine and health promotion during family medicine rotations is common practice in Thailand. Lastly, the findings were based on expert opinions about medical education that could not reflect the efficacy and effectiveness of clinical practices and patient outcomes.

\section{CONCLUSIONS}

This is the first study conducted at a national level in Thailand to identify ways to develop training programmes for PA counselling in undergraduate medical education. The definition and types of PA, the FITT principle and benefits of PA are essential training for medical students. Moreover, training in PA counselling skills should include general communication skills, counselling techniques and the 5 A's. Both intracurricular and extracurricular activities are required. Building collaborations between 
medical educators and health institutions, as well as implementing effective educational policies, are additional approaches that will be needed to inculcate PA counselling in clinical practice. Future research should focus on implementing training programmes for the undergraduate medical curriculum and to investigate the learning outcomes of medical students. In addition, there is a need to elucidate the clinical outcomes in patients that are the endpoints of the training.

Acknowledgements The authors wish to thank the participants for their cooperation, as well as Atchara Aidwang and Aekthawat Watthanachon for their support during this study.

Contributors AW, SW and TT initiated the concepts of the study. AW and PP designed the methodology. All the authors contributed to data collection and analysis. AW wrote the first draft of the manuscript and the rest of the team read and critically revised the draft. All the authors read and approved the final version of the manuscript.

Funding This study was granted by the Physical Activity Research Center (grant number: 60-00-05) and partially supported by the New Strategic Research (P2P) Project, Walailak University, Thailand.

Competing interests None declared.

Patient consent for publication Not required.

Ethics approval This study was approved by the Human Research Ethics Committee of Walailak University (protocol number: WUEC-18-039-01). The participation in this study was voluntary. All participants were asked to give informed consent via the online system by ticking the checkbox at the end of the information sheet.

Provenance and peer review Not commissioned; externally peer reviewed.

Data availability statement Data are available on reasonable request.

Open access This is an open access article distributed in accordance with the Creative Commons Attribution Non Commercial (CC BY-NC 4.0) license, which permits others to distribute, remix, adapt, build upon this work non-commercially, and license their derivative works on different terms, provided the original work is properly cited, appropriate credit is given, any changes made indicated, and the use is non-commercial. See: http://creativecommons.org/licenses/by-nc/4.0/.

\section{REFERENCES}

1. Vuori IM, Lavie CJ, Blair SN. Physical activity promotion in the health care system. Mayo Clin Proc 2013;88:1446-61.

2. Phillips EM, Kennedy MA. The exercise prescription: a tool to improve physical activity. PM R 2012;4:818-25.

3. Aittasalo M, Kukkonen-Harjula K, Toropainen E, et al. Developing physical activity counselling in primary care through participatory action approach. BMC Fam Pract 2016;17:141.

4. Orrow G, Kinmonth A-L, Sanderson S, et al. Effectiveness of physical activity promotion based in primary care: systematic review and meta-analysis of randomised controlled trials. BMJ 2012;344.

5. Hupin D, Edouard P, Gremeaux V, et al. Physical activity to reduce mortality risk. Eur Heart $J$ 2017;38:1534-7.

6. Lear SA, Hu W, Rangarajan S, et al. The effect of physical activity on mortality and cardiovascular disease in 130000 people from 17 highincome, middle-income, and low-income countries: the PURE study. The Lancet 2017;390:2643-54.

7. Pedersen BK, Saltin B. Exercise as medicine - evidence for prescribing exercise as therapy in 26 different chronic diseases. Scand J Med Sci Sports 2015;25 Suppl 3:1-72.

8. Russell E. Exercise is medicine. Can Med Assoc J 2013;185:E526.

9. Taylor D. Physical activity is medicine for older adults. Postgrad Med J 2014;90:26-32.

10. Hébert ET, Caughy MO, Shuval K. Primary care providers' perceptions of physical activity counselling in a clinical setting: a systematic review. Br J Sports Med 2012;46:625-31.
11. World Health Organization. Global action plan on physical activity 2018-2030: more active people for a healthier world. Geneva: World Health Organization, 2018.

12. Stoutenberg M, Stasi S, Stamatakis E, et al. Physical activity training in US medical schools: preparing future physicians to engage in primary prevention. Phys Sportsmed 2015;43:388-94.

13. Strong A, Stoutenberg M, Hobson-Powell A, et al. An evaluation of physical activity training in Australian medical school curricula. $J$ Sci Med Sport 2017;20:534-8.

14. Weiler R, Chew S, Coombs N, et al. Physical activity education in the undergraduate curricula of all UK medical schools: are tomorrow's doctors equipped to follow clinical guidelines? Br J Sports Med 2012;46:1024-6.

15. Wattanapisit A, Vijitpongjinda S, Saengow U, et al. Results from the medical school physical activity report card (MSPARC) for a Thai medical school: a mixed methods study. BMC Med Educ 2018;18.

16. Dacey ML, Kennedy MA, Polak R, et al. Physical activity counseling in medical school education: a systematic review. Med Educ Online 2014;19:24325.

17. Wattanapisit A, Tuangratananon T, Thanamee S. Physical activity counseling in primary care and family medicine residency training: a systematic review. BMC Med Educ 2018;18.

18. Sallis R. Exercise is medicine: a call to action for physicians to assess and prescribe exercise. Phys Sportsmed 2015;43:22-6.

19. Chisholm A, Ang-Chen P, Peters S, et al. Public health practitioners' views of the 'Making Every Contact Count' initiative and standards for its evaluation. J Public Health 2018;41.

20. Diamond IR, Grant RC, Feldman BM, et al. Defining consensus: a systematic review recommends methodologic criteria for reporting of Delphi studies. J Clin Epidemiol 2014;67:401-9.

21. O'Brien M, Shields C, Crowell S, et al. The effects of previous educational training on physical activity counselling and exercise prescription practices among physicians across nova Scotia: a cross-sectional study. Can Med Educ J 2018;9:e35-45.

22. Polak R, Pojednic RM, Phillips EM. Lifestyle medicine education. Am J Lifestyle Med 2015;9:361-7.

23. Phillips E, Pojednic R, Polak R, et al. Including lifestyle medicine in undergraduate medical curricula. Med Educ Online 2015;20:26150.

24. Mcleod P, Steinert Y. Twelve tips for curriculum renewal. Med Teach 2015;37:232-8.

25. Jadczak AD, Tam KL, Visvanathan R. Educating medical students in counselling older adults about exercise: the impact of a physical activity module. J Frailty Aging 2018;7:113-9.

26. Denniston C, Molloy E, Nestel D, et al. Learning outcomes for communication skills across the health professions: a systematic literature review and qualitative synthesis. BMJ Open 2017;7:e014570.

27. Deveugele M, Derese A, De Maesschalck S, et al. Teaching communication skills to medical students, a challenge in the curriculum? Patient Educ Couns 2005;58:265-70.

28. Haq C, Steele DJ, Marchand L, et al. Integrating the art and science of medical practice: innovations in teaching medical communication skills. Fam Med 2004;36 Suppl:S43-50.

29. Sicat BL, O'Kane Kreutzer K, Gary J, et al. A collaboration among health sciences schools to enhance faculty development in teaching. Am J Pharm Educ 2014;78:102.

30. Lobelo F, Duperly J, Frank E. Physical activity habits of doctors and medical students influence their counselling practices. $\mathrm{Br} J$ Sports Med 2009;43:89-92.

31. Stanford FC, Durkin MW, Stallworth JR, et al. Factors that influence physicians' and medical students' confidence in counseling patients about physical activity. J Prim Prev 2014;35:193-201.

32. Wattanapisit A, Fungthongcharoen K, Saengow U, et al. Physical activity among medical students in southern Thailand: a mixed methods study. BMJ Open 2016;6:e013479.

33. Van Mol C. Improving web survey efficiency: the impact of an extra reminder and reminder content on web survey response. Int J Soc Res Methodol 2017;20:317-27.

34. Thangaratinam S, Redman CWE. The Delphi technique. Obstet Gynecol 2005;7:120-5.

35. Hsu; CC, Sandford BA. The Delphi technique: making sense of consensus. PARE 2007;12.

36. Wattanapisit A, Vijitpongjinda S, Saengow U, et al. Development of a physical activity monitoring tool for Thai medical schools: a protocol for a mixed methods study. BMJ Open 2017;7:e017297. 